\title{
LAS CIENCIAS AMBIENTALES: UN ÁREA DEL CONOCIMIENTO PARA EL DESARROLLO DE ENFOQUES INTERDISCIPLINARIOS
}

\section{Environmental Sciences: A field of knowledge for the development of interdisciplinary approaches}

\author{
ENRIQUE JAVIER PEÑA S. ${ }^{1}$ JAIRO ROLDÁN CH. ${ }^{2}$ \\ 1Departamenteo de Biología, Universidad del Valle. ${ }^{2}$ Departamento de Física, Universidad del Valle.
}

E-mail: enrique.pena@correounivalle.edu.co

jairoroldan@gmail.com

\section{Resumen}

En el artículo se presentan algunas de las preguntas fundamentales en torno al medio ambiente, las cuales han llevado a muchos autores a señalar que, dada su complejidad, el estudio del medio ambiente no pertenece a ninguna ciencia o disciplina. Se discuten también varias propuestas interdisciplinarias de algunos autores en torno al estudio del medio ambiente. Con base en el análisis se propone entonces una serie conceptos acerca del medio ambiente que se consideran necesarios para abordar adecuadamente el conocimiento de nuestro entorno. Al final se lleva a cabo una discusión sobre las ciencias ambientales y el enfoque interdisciplinario.

Palabras claves: Medio ambiente, Ciencias ambientales, Interdisciplinariedad.

\section{Abstract}

In the article some of the fundamental questions about the environment are presented. Those questions have led many authors to point out that given its complexity the study of the environment does not belong to any science or discipline. Several interdisciplinary proposals of some authors on the study of the environment are also discussed. Based on the analysis, a series of concepts are then proposed which are considered as necessary for arriving to an adequate knowledge of our environment. At the end a discussion is conducted on environmental sciences and interdisciplinary approaches.

Keywords: Environment, Environmental sciences, Interdisciplinarity. 


\section{INTRODUCCIÓN}

Como parte de la experiencia cotidiana, la población mundial sufre las consecuencias negativas de la destrucción de la naturaleza. Es una situación que el colectivo de la gente no repara en términos del impacto que esto tiene en la salud, la biodiversidad, las actividades productivas locales, la movilidad social y la valoración cultural de los recursos naturales. Por tal razón desde la óptica de las ciencias ambientales se plantean algunas líneas generales en torno al desequilibrio ecológico que actualmente se vive y a las posibilidades de construir una racionalidad alterna, capaz de vincular los procesos naturales y sociales, incorporar la práctica de la interdisciplinariedad y generar las condiciones necesarias para un nuevo enfoque de aprendizaje.

Hechos como la contaminación y el agotamiento de los mantos acuíferos; la degradación de la calidad del aire; la erosión y pérdida de fertilidad de los suelos; el incremento de los residuos urbanos y los desechos tóxicos industriales; la contaminación auditiva en las ciudades; el incremento en el desequilibrio entre la producción de entropía y las tasas de producción de biomasa; el calentamiento global; la existencia de venenos radiactivos y la pérdida acelerada de biodiversidad son sólo algunas de las consecuencias negativas del comportamiento irracional de la sociedad frente a la naturaleza.

\section{Propuestas interdisciplinarias en torno al estudio del ambiente}

Muchos autores han señalado que, dada su complejidad, el estudio del medio ambiente no pertenece a ninguna ciencia o disciplina. En un principio se creía que la ecología y la biología eran las ciencias más adecuadas para estudiar las interrelaciones de los seres vivos con su entorno natural; sin embargo, pronto se comprendió que la coevolución de las culturas y la naturaleza, así como los efectos antropogénicos sobre ésta, no podían ser explicados, al menos no exclusivamente, desde las llamadas "ciencias duras".

Llegó a entenderse que la capitalización de los recursos naturales, la in-sustentabilidad del uso de la energía fósil, el aumento del efecto invernadero, la pérdida de biodiversidad y la disipación creciente de masa y energía en el planeta, debían ser explicados desde una óptica social, capaz de vincular las condiciones naturales del entorno con su utilización productiva y la valoración cultural que de ellas se tiene. Así, se fueron delineando preguntas de investigación y construyendo objetos de estudio que no pertenecían a ninguno de los campos temáticos tradicionales de las distintas ciencias. Preguntas como: ¿puede el mercado ser un instrumento eficaz para la conservación de la naturaleza?, ¿es posible lograr un equilibrio entre la ineluctable producción de entropía y las tasas de producción de biomasa?, o ¿qué significa lograr un desarrollo sustentable para este planeta?, han cobrado cada vez más importancia.

Interdisciplinariedad sin duda enriquecedora, pero también altamente compleja, dada la enorme red de discursos divergentes que en ella tienen lugar. Concepto, el de interdisciplinariedad, en el que se encuentran al menos dos acepciones que es necesario precisar: 1) contribución de distintas disciplinas, con sus métodos, teorías y perspectivas propias, para construir un nuevo objeto de conocimiento; 2) integración de distintos paradigmas del conocimiento, mediante la problematización y desconstrucción de sus presupuestos epistemológicos fundamentales. Ambas parecen correctas, motivo por el cual se las menciona; sin embargo, en la segunda acepción, contrario a la primera, existe un cuestionamiento profundo de los presupuestos fundamentales de las distintas ciencias, lo que implica no sólo contribuir, desde distintas perspectivas, a la construcción de un nuevo objeto de conocimiento, sino desconstruir las bases desde las que se parte.

En el caso de la transdisciplinariedad, una acepción del concepto es que hace referencia a la trascendencia de la interacción entre las disciplinas, lo que implica, en los métodos científicos y en la actitud pedagógica, la superación de las fronteras entre las ciencias, sin oponer unas a otras, mediante la articulación de los saberes y la circulación de conceptos y valores. Temas como el saber ambiental, la transgénesis, el riesgo (ecológico, biotecnológico, nuclear) y la desterritorialización de las relaciones sociales, son considerados de frontera.

Se presentan a continuación algunas propuestas de articulación interdisciplinaria de distintos investigadores, los cuales, desde puntos de vista diversos, analizan las diversas variables de la complejidad ambiental, caracterizándola como algo distinto del estudio ecológico del entorno.

Enrique Leff, coordinador de la Red de Formación Ambiental para América Latina y el Caribe, miembro del Programa de Naciones Unidas para el Medio Ambiente (PNUMA), expone en distintos trabajos la interrelación que para el estudio de la complejidad ambiental existen entre la biotecnología, las ciencias etnológicas, la sociodemografía, la ecología (política, productiva, social), la economía (ecológica, política del ambiente), la educación, la historia y la pedagogía ambientales, la ética (de la interculturalidad, para la sustentabilidad, por la vida), el psicoanálisis y la sociología del conocimiento (Leff 2001, 2002a, 2002b). 
Para este autor, por mencionar un ejemplo, los principios éticos no son incompatibles con las ideas de desarrollo, de racionalidad y de la diferencia, pero sí con ciertas concepciones modernas de la moral y de la ciencia: aquéllas sustentadas en los principios del individualismo, la competencia, la exclusión de los saberes no científicos, el utilitarismo de la tecnología y el antropocentrismo. Al respecto, en el trabajo "Ética por la vida. Elogio de la voluntad de poder" expone que:

"La ética del conocimiento para el desarrollo sustentable es una ética de los límites y del sin sentido a los que ha llegado un modelo de conocimiento construido en la Modernidad, centrado en la visión economicista del mundo, la mercantilización de la cultura y una ciencia y tecnología concebidas para el dominio de la naturaleza. La ética del saber ambiental lleva a desmontar la epistemología que ha cosificado, objetivado y alienado al mundo y construir un saber emancipatorio que pueda conjugar la sustentabilidad y la solidaridad; que lleve a una reterritorialización de las ideas originadas en el océano de la complejidad. Y eso es una cuestión del ser y del tiempo." (Leff 2002a).

Se trata de cuestionamientos éticos y filosóficos que rebasan por mucho teorías reduccionistas centradas en el individuo, en la cuantificación de las reservas naturales, en la valoración del potencial productivo de éstas o en la interacción simple entre sociedad y naturaleza; y que, por el contrario, involucran asuntos existenciales valorados a la luz de consideraciones espacio-temporales. Es, como el autor lo dice, "una cuestión del ser y del tiempo".

Otro autor, Arturo Escobar, profesor de la Universidad de Carolina del Norte en Chapel Hill, se enfoca en la antropología, particularmente en la teoría social del postestructuralismo, la cual coloca en la base del conocimiento y de la dinámica de lo social al lenguaje y la significación. Para ello habla de la antropología de la naturaleza, de la tecnociencia, de los movimientos sociales, del desarrollo y del lugar, además de la ecología política (Escobar 1999). De éstas, rescatamos tres: la antropología del desarrollo, centrada en el análisis crítico del desarrollo como discurso y como práctica cultural; la antropología de la naturaleza, enfocada en el estudio de las distintas construcciones de la conservación de la biodiversidad en las regiones, y la antropología ecológica, interesada en la documentación etnográfica de modelos locales de naturaleza (Escobar 1999).

Antonio Aledo y José Andrés Domínguez optan por la sociología medioambiental y los diversos estudios que ésta puede ofrecer (Aledo \& Domínguez 2001). Guillermo Castro, investigador asociado del Centro de Estudios Latinoamericanos "Justo Arosemena", en Panamá, analiza la vinculación entre la economía, la historia (natural, económica, social) y la sociodemografía (Castro 1996).

Por último, hacemos referencia sólo a otros tres autores: Louis Lemkow, Moacir Gadotti y Joan Martinez-Alier. El primero, catedrático de la Universitat Autónoma de Barcelona, se enfoca en la sociología ambiental, aunque también considera a la antropología (ecológica, social); la ecología (cultural, humana, sistémica, social del riesgo); la geografía humana; el pensamiento socioambiental y la sociobiología (Lemkow 2002). Para este autor, los hechos que explican la interdisciplinariedad en el estudio de las cuestiones ambientales, son distintos en particular a los de las ciencias sociales, cuyo despegue, o incorporación social de la variable ambiental, la sitúa en la década de 1960.

“Su aparición puede explicarse por razones diversas, y no es la menos importante la que relaciona dicha aparición con una nueva construcción social de la ciencia, de la tecnología $\mathrm{y}$ de los riesgos ambientales en un mundo dominado por la inseguridad generada por la Guerra Fría y el peligro de una conflagración nuclear. La inseguridad estaría también relacionada con cambios objetivos en el alcance y naturaleza de la degradación ambiental que, como han desvelado algunos estamentos científico-técnicos, serían susceptibles de provocar notables impactos sobre la salud humana y sobre el equilibrio de los ecosistemas. En este contexto, tuvo también gran importancia el papel que jugaron los movimientos emergentes que denunciaban los peligros de las agresiones ambientales perpetradas por economías productivistas (tanto capitalistas como socialistas). Por otra parte, la crisis de la propia sociología, durante la década de los sesenta, constituye otro factor de tipo contextual que contribuyó a la proliferación de nuevos enfoques y paradigmas." (Lemkow 2002).

La sociología ambiental tiene como fuentes, directas o indirectas, los avances científico-tecnológicos, las confrontaciones políticas internacionales, la amenaza de la guerra nuclear, el incremento en la degradación ecológica, el desarrollo de movimientos sociales emergentes y la "crisis teórica" de la sociología.

Por su parte, Moacir Gadotti, director del Instituto Paulo Freire, comenta en sus estudios acerca de la educación (sustentable, del futuro); de la filosofía de la naturaleza y de la pedagogía (crítico-liberadora, de la praxis, de la tierra, dialógica, ecológica) (Gadotti 2002), que la educación, vista como una práctica liberadora, cambia las concepciones individuales y sociales, $\mathrm{y}$, potencialmente, permite la transformación total de la sociedad. 
Para algunas corrientes sociales toda conducta humana es, en realidad, producto de una educación que comienza en el momento del nacimiento de las personas, hecho que significa que la labor educativa es un acto de suma importancia para la conservación de la naturaleza. Gadotti (2002), por ejemplo, propone construir una ecopedagogía vinculada con lo que se conoce como pedagogía del oprimido.

Vinculación, la del ambiente con las condiciones de vida de las personas (opresión, exclusión y pobreza), que también es analizada por Joan Martinez-Alier (2002), miembro del comité científico de la Agencia Ambiental Europea, quien, en distintos trabajos, ha argumentado acerca del ambientalismo de los pobres ("The environmentalism of the poor"). La distribución de los conflictos ecológicos, la insustentabilidad urbana, la defensa del ambiente en áreas urbanas y rurales por parte de la gente pobre, la conservación de los derechos de propiedad comunitaria, la biopiratería en la agricultura, los derechos de propiedad intelectual, la propuesta de la agroecología, la seguridad alimentaria de los pueblos y los conflictos ecológicos causados por el crecimiento de las ciudades, son algunos de los tópicos que este autor maneja en sus trabajos.

Un punto adicional que maneja Martinez-Alier (2002) es que raramente los agentes de estos conflictos se asumen ellos mismos como ambientalistas. Situación que se puede explicar debido a que estas personas no buscan la protección integral del ambiente a partir de concepciones teóricas que sustenten sus acciones. Por el contrario, parten de lo que consideran derechos adquiridos y estilos de vida propios.

Los autores mencionados son todos de reconocido prestigio y sus ideas permiten argumentar acerca de la importancia de la inter y la transdisciplinariedad para el estudio de fenómenos complejos como lo es el ambiente. Sus análisis ayudan también a generar las condiciones necesarias para un nuevo enfoque de aprendizaje.

A continuación se propone una serie conceptos acerca del medio ambiente que consideramos necesarios para abordar adecuadamente el conocimiento de nuestro entorno.

1. El ambiente es un constructo gnoseológico y epistemológico que hace referencia a la externalidad, a lo que está fuera, tanto de los sistemas como de los campos de conocimiento.

2. Es importante reconocer que el ambiente ha sido tradicionalmente el dominio del no saber, de lo no pensado. Puede afirmarse entonces que el ambiente es un concepto que articula lo no pensado.
3. El ambiente es el dominio generalizado de las relaciones sociedad-naturaleza.

4. El ambiente es el campo material y abstracto de las relaciones cultura, población, naturaleza y trabajo.

La consideración epistemológica del ambiente como externalidad, implica el análisis profundo de las teorías referentes al conocimiento válido y de los procesos de su validación. En una óptica crítica, conlleva la significación de las estrategias de poder en el saber, teóricamente definida como epistemología política.

Las relaciones sociedad-naturaleza, con todo lo que ello implica: sistemas de significados, valoración cultural, estrategias de apropiación, utilización productiva, distribución y comercio, conflictos ecológicos, etcétera, son también parte de lo que es el ambiente, en términos de que todos estos elementos son parte de este sistema de relaciones tradicionalmente ignorado por la ciencia.

\section{Las ciencias ambientales y el enfoque interdisciplinario}

Muchas son las cuestiones referentes al medio ambiente y a la interacción de las disciplinas sociales y naturales, que es necesario abordar desde ópticas interdisciplinarias. Al respecto, es importante argumentar acerca de la importancia de la sociología para explicar las causas y los efectos que tienen las acciones humanas en la naturaleza y las formas en que ésta impacta las formas de ser y estar en el mundo.

Tradicionalmente se concibe la sociología como la ciencia encargada de estudiar las estructuras y sistemas sociales, y la forma en que interactúan con el individuo y los grupos sociales. Por definición parece excluir la naturaleza, debido a que la evolución de ésta, mediante innumerables ciclos biogeoquímicos que le son inherentes, no depende de las actividades humanas, por lo que es un proceso asocial. Cuestión que no significa ignorar que la naturaleza es permanentemente afectada por procesos antrópicos, como el consumo -endosomático y exosomático - de materia y energía. Sin embargo, la sociología, como ciencia que estudia la producción y reproducción de lo social y de la sociedad, suele considerar al ambiente como sinónimo de naturaleza y a ésta como parte del entorno donde las personas realizan sus actividades.

Esta idea del entorno, concebido como lo que rodea físicamente a algo, suele ser considerada en estudios de sociología comprensiva, al razonar los escenarios en los que se desenvuelven las acciones provistas de sentido y las relaciones sociales que de éstas se desprenden. Su 
importancia radica en la ubicación espacio-temporal, donde las personas realizan sus acciones.

Por ejemplo, un agricultor tiene que considerar el clima como una variable importante para saber qué y cuándo cultivar. Sin embargo, su reflexión no parte de una conciencia ecológica que busca lograr una gestión ambiental sustentable, sino de la necesidad de escoger la forma más adecuada de producir con el fin de obtener mayores cosechas $\mathrm{y}$, potencialmente, mejores ganancias en el mercado. La construcción de su conocimiento, aunque valora las condiciones específicas del aquí y del ahora, está posiblemente sustentada en una lógica de la ganancia que poco tiene que ver con la sustentabilidad del hábitat en el que cultiva. Por ello es que críticamente se afirma que la falta de ambiente en el conocimiento se visibiliza después en la generación de problemas ecológico-ambientales.

Desde la óptica de la racionalidad económica dominante, las ideas tradicionales de lo que constituye cultura y desarrollo implican ganarle terreno a la naturaleza, más que la convivencia armoniosa con ella. La modificación, por tanto, de los patrones de producción y consumo, depende de las representaciones culturales que se tengan de lo que es común, público y privado, desde la tierra, hasta los animales que habitan en ella. Las reservas de biodiversidad, por ejemplo, contrario a la lógica capitalista, significan que éstas tienen un valor más allá de la propiedad privada.

¿Cuánto vale el último delfín de la Tierra? Sería la pregunta que uno podría hacerse si todo estuviera en venta. Afortunadamente no es así, aunque en los hechos muchas son las especies en peligro de extinción por la avaricia humana. No planteamos, en este sentido, un problema de desequilibrio ecológico, al menos no únicamente, sino de insustentabilidad en la racionalidad económica dominante.

La agroecología, la agroforestería, la bioética, la búsqueda del equilibrio entre la producción de entropía y las tasas de producción de biomasa, la contribución al ciclo del agua, la conversión del planeta en un gran colector solar, la conservación de corredores biológicos, la crítica termodinámica a la economía, los cultivos integrados, el desarrollo limpio, el ecodesarrollo, la ecología de la acción, la economía ecológica, la educación ecológica popular, el reconocimiento de la deuda ecológica, entre otras muchas acciones, son algunas de las propuestas que se han planteado para revertir los procesos de deterioro ambiental. Para lograrlo es importante reconocer que tradicionalmente el lugar del ambiente ha sido el no saber, lo no pensado, lo que significa al ambiente como un constructo gnoseológico y epistemológico que hace referencia a la externalidad, lo que está fuera, de los sistemas y de los campos de conocimiento.
Es, como se señaló antes, un concepto articulador de lo no pensado.

La interdisciplinariedad desde un enfoque ambiental tiene entonces como uno de sus propósitos facilitar la reapropiación de la naturaleza, no en términos de la explotación de la que puede ser objeto, sino de la valoración de su potencial ecológico productivo. Cuestión ya considerada por los saberes tradicionales, precisamente cuando hablaban del principio de autogestión de las sociedades agrarias y de la productividad primaria de los ecosistemas naturales. La propuesta, en síntesis, es la integración del conocimiento basado en los principios de la interdisciplinariedad y sustentada en el estudio de las ciencias ambientales como una nueva área del conocimiento.

\section{REFERENCIAS}

ALEDO, A. \& DOMÍNGUEZ, J. A. 2001. Sociología ambiental. Grupo Editorial Universitario, España. 462 pp.

ALIER-MARTINEZ, J. 2002. The environmentalism of the poor (a report for UNRISD for the WSSD), University of Witswatersrand, 30 de agosto, versión en archivo digital 159.

CASTRO, G. 1996. Naturaleza y sociedad en la historia de América Latina. Centro de Estudios Latinoamericanos "Justo Arosemena", Panamá. 351 pp.

ESCOBAR, A. 1999. El final del salvaje. Naturaleza, cultura y política en la antropología contemporánea. Manuela Álvarez, Santafé de Bogotá, Instituto Colombiano de Antropología, Centro de Estudios de la Realidad Colombian. 418 pp.

GADOTTI, M. 2002. Pedagogía de la Tierra. Editorial Siglo XXI, México. 195 pp.

LEFF, E. 1994. Ciencias sociales y formación ambiental, Barcelona, Gedisa, Centro de Investigaciones Interdisciplinarias en Humanidades. Universidad Nacional Autónoma de México. 321 pp.

LEFF, E. 2000a. La complejidad ambiental Editorial Siglo XXI, México. 314 pp.

LEFF, E. 2000b, Los problemas del conocimiento y la perspectiva ambiental del desarrollo. Editorial Siglo XXI, México. 409 pp. 
LEFF, E. 2010. Ecología y capital. Racionalidad ambiental, democracia participativa y desarrollo sustentable. Editorial Siglo XXI, México. 437 pp.

LEFF, E. 2002a. Ética, vida, sustentabilidad. Editorial Siglo XXI, México 331 pp.

LEFF, E. 2002b. Saber ambiental. Sustentabilidad, racionalidad, complejidad, poder. Editorial Siglo XXI, México. 414 pp.

LEMKOW, L. 2002. Sociología ambiental: Pensamiento socioambiental y ecología social del riesgo. Editorial Icaria, Barcelona. 232 pp. 